\title{
Editorial: Dynamic Scheduling Problems
}

\author{
Alessandro Agnetis ${ }^{1} \cdot$ Stanisław Gawiejnowicz ${ }^{2}$ (D) $\cdot$ Bertrand Miao-Tsong Lin $^{3}$. Gur Mosheiov ${ }^{4}$
}

Accepted: 16 September 2020 / Published online: 24 October 2020

(c) Springer Science+Business Media, LLC, part of Springer Nature 2020

This special issue of the Journal of Scheduling contains selected papers submitted in response to an open call for papers announced after the Second International Workshop on Dynamic Scheduling Problems (IWDSP 2018), held in June 2018, at the Faculty of Mathematics and Computer Science, Adam Mickiewicz University, Poznań, Poland. It was the second IWDSP workshop, following the IWDSP 2016 workshop held at the same faculty two years earlier. The third workshop in the series, IWDSP 2020, was to be held in June 2020, but in view of the COVID-19 pandemic it has been postponed until 2021.

The IWDSP 2018 workshop, similarly to its predecessor, focused on dynamic scheduling problems, in which job processing times, machine speeds and other parameters of the problems are variable and dynamically change in time. The organization of both these events was supported by Adam Mickiewicz University, Poznan. Extended abstracts of the papers presented at the workshops were published by the Polish Mathematical Society; their electronic versions are available at the websites https://iwdsp2016.wmi.amu.edu.pl and https://iwdsp2018.wmi.amu.edu.pl.

We received 18 submissions to this special issue, written by authors from Belarus, the People's Republic of China, Israel, Italy, Poland, the Russian Federation, Switzerland and

Stanisław Gawiejnowicz

stgawiej@amu.edu.pl

Alessandro Agnetis

agnetis@dii.unisi.it

Bertrand Miao-Tsong Lin

bmtlin@mail.nctu.edu.tw

Gur Mosheiov

msomer@huji.ac.il

1 Dipartimento di Ingegneria dell'Informazione, Università degli Studi di Siena, Siena, Italy

2 Faculty of Mathematics an Computer Science, Adam Mickiewicz University, Poznań, Poland

3 Institute of Information Management, National Chiao Tung University, Hsinchu, Taiwan

4 The Jerusalem School of Business Administration, The Hebrew University of Jerusalem, Jerusalem, Israel the United Kingdom. In order to ensure the highest quality of papers, all submissions have undergone the standard peer reviewing process of the Journal of Scheduling. As a result of this process, 10 submissions were accepted for publication.

The papers included in this special issue concern scheduling deteriorating jobs, scheduling with learning effects, energy-efficient scheduling, scheduling in data-gathering networks, scheduling under uncertainty, scheduling with job rejection, and dynamic pickup and delivery problems with time windows. They span a wide range of applications of dynamic scheduling problems in production environments, manufacturing systems and service management. The content of the papers is as follows.

In the paper 'Scheduling in data gathering networks with background communications', Berlińska considers a scheduling problem in star data gathering networks with background communications. She proves strong $\mathcal{N} \mathcal{P}$-hardness of the non-preemptive variant of the problem, shows how to solve its preemptive counterpart in polynomial time, and proposes an exact algorithm, three heuristics and four local search algorithms for the non-preemptive variant.

In the paper 'New results for an open time-dependent scheduling problem', Gawiejnowicz and Kurc prove a new necessary condition of schedule optimality for the still open single machine time-dependent scheduling problem of minimizing the total completion time for a set of linearly deteriorating jobs with unit basic processing times. This condition implies the improvement of the previous bound on the number of all possible optimal schedules for the problem by a multiplicative factor which is proportional to the reciprocal of the square root of the number of jobs.

In the paper 'A technical note: FPTASes for minimizing makespan of deteriorating jobs with non-linear processing times', Halman constructs a fully polynomial time approximation scheme for a single machine problem of scheduling deteriorating jobs with nonlinear processing times. This scheme applies a new technique for the development of approximation schemes called K-approximation sets and functions, and is faster than the fastest scheme proposed for 
the problem earlier by a factor proportional to the cube of the number of jobs.

In the paper 'A periodic approach to dynamic pickup and delivery problems with time windows', Karami, Vancroonenburg and Vanden Berghe propose a mixed integer linear programming model and a two-step scheduling heuristic for dynamic pickup and delivery problems with time windows. This heuristic uses a buffering strategy-based periodic optimization approach, takes into account request urgency levels, and has only a small overhead.

In the paper 'Approximation algorithms for energy efficient scheduling for parallel jobs', Kononov and Kovalenko consider homogeneous job scheduling on speed-scalable processors. They develop constant-factor approximation algorithms for preemptive agreeable jobs without migration and a 3-approximation algorithm for non-preemptive agreeable jobs with unit-work operations.

In the paper 'Less is more: variable neighbourhood search for integrated production and assembly in smart manufacturing', Lu, Pei, Qian, Mladenovic and Pardalos propose a variable neighborhood search heuristic for an integrated production and assembly scheduling problem with serial batching and the effects of job deterioration and learning.

In the paper 'Flow shop scheduling with learning-effect and job-rejection', Mor, Mosheiov and Shapira present pseudo-polynomial dynamic programming algorithms for a proportionate flow shop scheduling problem with a learning effect, job rejection and the criteria of maximum completion time, total completion time and total load. The proposed algorithms are very efficient and solve instances with 80 jobs in no more than $5 \mathrm{~ms}$.

In the paper 'Scheduling jobs with a V-shaped timedependent processing time', Sedding constructs an FPTAS for an agreeable case of a single machine time-dependent scheduling problem with convex V-shaped job processing times and the maximum completion time criterion. He also identifies a few polynomial cases of the problem.
In the paper 'On the complexity of finding minimax regret solution for two-machine flow shop problem under the interval uncertainty', Shafransky and Shinkarevich apply a recently proposed alternative scheme for proving $\mathcal{N} \mathcal{P}$. hardness of optimization problems, and prove that the two-machine flow shop problem under interval uncertainty of job processing times is $\mathcal{N} \mathcal{P}$-hard and that it cannot be approximated in polynomial time.

Finally, in the paper 'Refined conditions for V-shaped optimal sequencing on a single machine to minimize total completion time under combined effects', Soper and Strusevich propose a method for checking whether a given optimal sequence is V-shaped, and apply it to several single-machine scheduling problems with various positional and cumulative effects.

We express our sincere thanks to Michael L. Pinedo, former Editor of the Journal of Scheduling, for the idea of the special issue and for his remarks and suggestions, and to Edmund Burke, Editor-in-Chief of the journal, who accepted our proposal and trusted our recommendations. We thank the referees who have provided high-quality reviews despite the time-demanding engagements we are all committed to.

Research on scheduling problems with dynamically changing parameters is still in progress. We hope that this special issue will attract new researchers to the fascinating world of dynamic scheduling problems.

Publisher's Note Springer Nature remains neutral with regard to jurisdictional claims in published maps and institutional affiliations. 